\title{
«Creutes » et réseaux de défenses dans les paysages de guerre, exemple du Mont-Chatte (Hermonville, France)
}

Alain Devos, Sébastien Laratte, Nicolas Bollot et G. Fronteau

\section{OpenEdition}

\section{Journals}

Édition électronique

URL : https://journals.openedition.org/geohist/646

DOI : 10.4000/geohist.646

ISSN : 2264-2617

Éditeur

Association française de la Revue de géographie historique

Référence électronique

Alain Devos, Sébastien Laratte, Nicolas Bollot et G. Fronteau, « «Creutes » et réseaux de défenses dans les paysages de guerre, exemple du Mont-Chatte (Hermonville, France) », Revue de géographie historique [En ligne], 14-15 | 2019, mis en ligne le 28 mai 2018, consulté le 12 juin 2021. URL : http:// journals.openedition.org/geohist/646 ; DOI : https://doi.org/10.4000/geohist.646

Ce document a été généré automatiquement le 12 juin 2021.

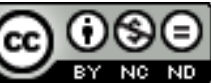

$\mathrm{Ce}(\mathrm{tte})$ œuvre est mise à disposition selon les termes de la Licence Creative Commons Attribution -

Pas d'Utilisation Commerciale - Pas de Modification 4.0 International. 


\title{
«Creutes » et réseaux de défenses dans les paysages de guerre, exemple du Mont-Chatte (Hermonville, France)
}

\author{
Alain Devos, Sébastien Laratte, Nicolas Bollot et G. Fronteau
}

Cet article s'intègre dans le programme de recherche Émergence Impact 14-18 porté par l'EA 3795 - GEGENAA et financé par la région Grand Est. Les auteurs tiennent à remercier l'association de la Cote 108, de la Main de Massiges et du Souvenir de Sommepy-Tahure ainsi que les musées de Meaux et de la Pompelle.

\section{Problématique et méthodes}

\section{A. Problématique}

1 La ligne de front de la Grande Guerre, trop souvent représentée sous la forme d'une ligne continue de $750 \mathrm{~km}$, des Flandres à la Suisse, cache une réalité géographique bien plus complexe. En effet, l'analyse spatiale des Plans Directeurs des Groupes de Canevas de Tirs des Armées (GCTA; Combaud et al., 2016), ou l'approche archéologique (Brenot et al., 2017) montrent que de part et d'autre de cette ligne, des réseaux de défense se font face, composées de positions et de lignes de tranchées, reliées par des boyaux, sur une profondeur de front allant de 10 à $40 \mathrm{~km}$ (Devos et al., 2015 ; Taborelli et al., 2018 ; Taborelli, et al., 2017a ;Taborelliet al., 2017b).

2 L'organisation spatiale de ces réseaux de défense dépend des potentialités topographiques, géomorphologiques, hydrogéologiques, des replis stratégiques et des paramètres militaires comme les crêtes militaires ou les portées de tir d'artillerie (Devos, et al., 2015 ; Villate, 1925). Ainsi, le front traverse plusieurs Pays géographiques, marqués à la fois par des potentialités et contraintes variées entre les plaines des Flandres, le Massif Vosgien, en passant par le relief de côtes du Bassin de Paris(Devos, et 
al., 2018). Dans la traversée des pays calcaires (calcaires tertiaires, craies secondaires, calcaires du Jurassique), de nombreuses cavités sont investies, réaménagées, réhabilitées afin d'être intégrées aux réseaux de défense (Laforgerie et Weiss, 2013 ; Weiss, 2016). C'est le cas pour d'anciennes crayères dans la région d'Arras (Bergerat et al., 2015; Prilaux et Bossut, 2018), mais plus particulièrement pour les carrières souterraines ou «creutes »du Noyonnais et du Soissonnais (Lachaux, 2005, 2008) et dans le secteur de Reims (Sosson et al., 2006).

3 L'objectif de cette contribution est double. Il s'agit d'une part de comprendre comment les carrières souterraines préexistantes sont intégrées aux réseaux de défense et d'autre part, d'apprécier les morphologies associées à leurs occupations par les militaires durant le conflit. En effet, si les polémo-formes généralement observées sous forêt (Amat, 2015; Taborelli, et al.2017b), sont nombreuses et très diversifiées, elles restent néanmoins peu étudiées dans le cadre de la guerre des mines (Brenot et al., 2017 ; Taborelli, et al., 2017a) et du monde souterrain pourtant largement impacté par la guerre (Triolet et Triolet, 2011).

Du Chemin des Dames, au secteur de Reims, la ligne de front jalonne les plateaux tertiaires, armés par les Calcaires grossiers du Lutétien à la craie de Champagne (Fig. 1). Ces calcaires du Lutétien moyen affleurent dans la partie sommitale des versants des nombreuses vallées qui découpent le plateau (Aisne, Ailette, Vesle, Ardre), mais sont recouverts par les Marnes et caillasses du Lutétien supérieur, représentées par des alternances de bancs décimétriques de marnes et de calcaires argileux ou sublithographiques. Ces calcaires furent exploités depuis l'Antiquité en carrières à ciel ouvert, en affleurement en bordure des plateaux, puis dans les creutes sous la couverture du Lutétien supérieur (Fronteau et al., 2014a). Ces dernières regroupent les habitats troglodytiques des "villages balcons" adossés au front de taille et de véritables carrières souterraines de plus grande ampleur, d'une centaine de mètres de développement à plusieurs hectares d'emprise au sol. Elles sont progressivement abandonnées et reconverties en champignonnières durant le XIX ${ }^{e}$ siècle avant d'être réaménagées en abris par les militaires durant la Grande Guerre. Après-guerre, dans le cadre de la reconstruction de la ville de Reims et de la restauration des monuments historiques, la recherche de " pierres équivalentes » s'est avérée difficile (Dolfini, 1920), et la majeure partie des carrières souterraines est tombéedans l'oubli. 
Document 1 : Carte de localisation du site d'étude et des creutes citées.

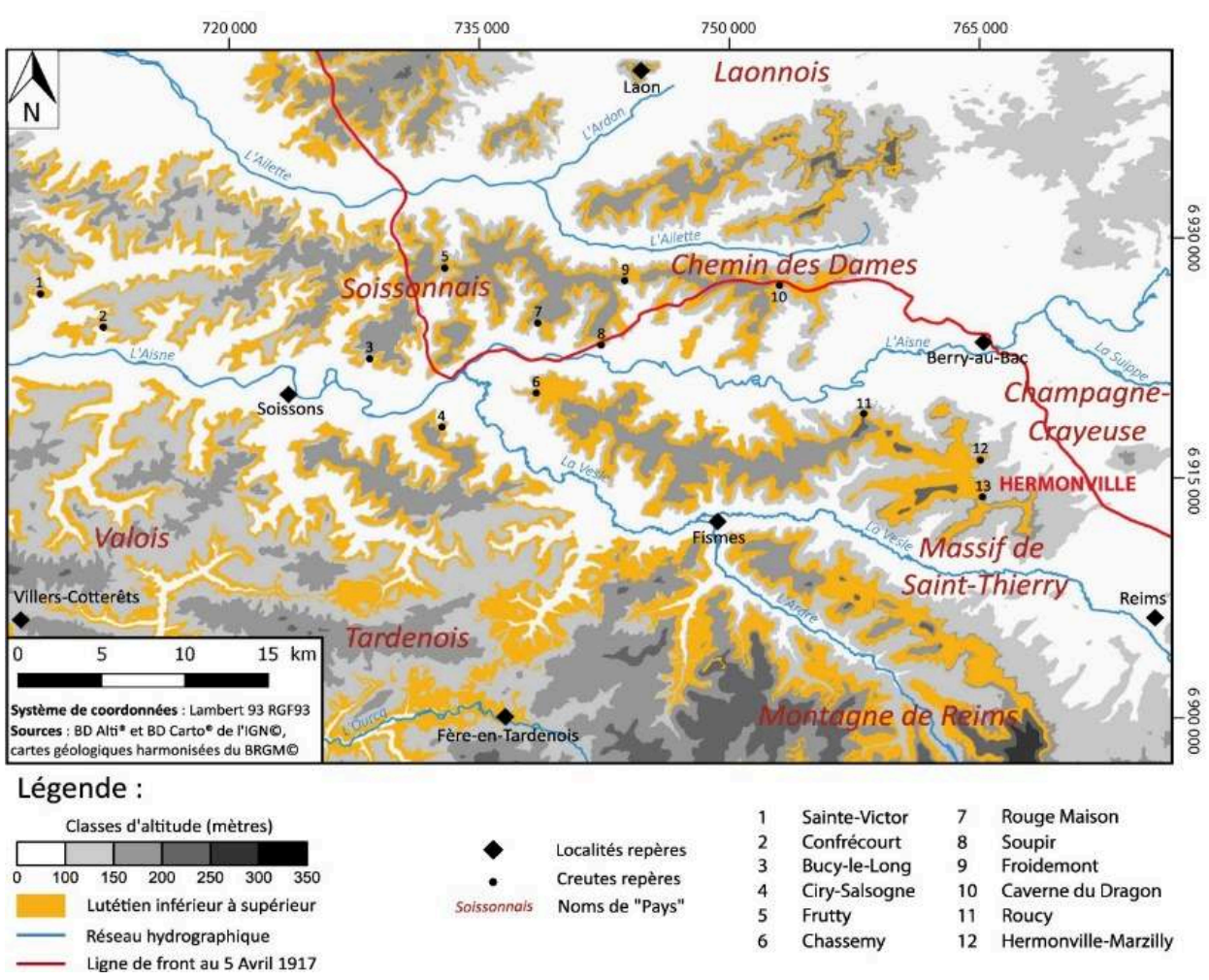

\section{B. Méthodes}

5 Le site du « Rougemont » ou " Mont Chatté »à Hermonville a été choisi comme zone atelier, car il s'inscrit dans le district carrier du massif forestier de Saint-Thierry ou «Petite Montagne de Reims » (Fronteau et al., 2014a). Il regroupe plusieurs carrières de pierre, à ciel ouvert et souterraines dans les calcaires du Lutétien. C'est un éperon de la côte d'Ile de France dominant Reims, inscrit dans l'entonnoir de percée cataclinale de la Vesle, et la plaine crayeuse de Champagne.

La première mention d'exploitation dans les environs d'Hermonville qui nous est parvenue date du XIV ${ }^{\mathrm{e}}$ siècle (Fronteau et al., 2014 b), celle-ci mentionnant des carrières déjà préexistantes dans ce secteur. Au cours des $\mathrm{XVI}^{\mathrm{e}}$ et XVII ${ }^{\mathrm{e}}$ siècles, les carrières exploitées semblent plutôt être localisées en vallée de la Vesle, mais le secteur d'Hermonville connaît une seconde grande phase d'activité aux XVIII ${ }^{e}$ et XIX ${ }^{\mathrm{e}}$ siècles. Ainsi sur la commune d'Hermonville en 1852, on dénombre 50 carrières souterraines occupant 105 ouvriers (Fronteau et al., 2014a). Mais avec la concurrence de pierres allogènes inhérente au développement $\mathrm{du}$ chemin de fer et aux voies navigables, les carrières sont progressivement abandonnées et/ou recyclées en champignonnières à la fin du XIX ${ }^{e}$ siècle. Durant la Grande Guerre, elles sont réhabilitées et réaménagées par l'Armée française en abris (Fig. 2). Le site présente de bonnes potentialités militaires pour l'observation du réseau de défense ennemi, pour l'artillerie et le cantonnement. Dans le cadre de l'offensive Nivelle du 16 avril 1917 au Chemin des Dames, des batteries de canons sont effectivement déployées sur l'éperon du Rougemont pour couvrir la zone de front entre Berry-au-Bac et Reims dans la vallée de la Loivre. Les observatoires du Rougement sont en liaison téléphonique avec plusieurs batteries lourdes dont celle 
de la casemate du Godat. La tour d'un moulin à vent ruiné sur le plateau sert de poste d'observation, les creutes sont électrifiées, ventilées puis aménagées pour le casernement. Des forages, des tunnels de liaison entre les carrières et des accès aux tranchées sont creusés. L'habitabilité, la protection face aux intempéries et aux bombardements sont soulignées par les poilus dans leurs écrits: «Creusées dans les profondeurs de la montagne, sinueuses comme des entrailles, se nouant, se dénouant, se confondant en de vastes places, pullulantes de zouaves accrochésen grappes sur lesbat-flancs, sur les couchettes disparaissant dans les hauteurs des parois, les champignonnières d'Hermonville constituaient un abri absolument remarquable contre les bombardements de l'ennemi » (Hanin, 2014).

7 Document 2 : Photographies aux champignonnières d'Hermonville (24/05/1918 BDIC La Contemporaine) A. Creusement d'une galerie d'accès de $20 \mathrm{~m}$ au perforateur (réf.4454), B. galerie d'accès coffrée de $17 \mathrm{~m}$ (réf.4448), C. galerie servant de chambrée (réf.4456), D. ventilateur et transport de pierres sur voie de $60 \mathrm{~cm}$ (réf.4451).
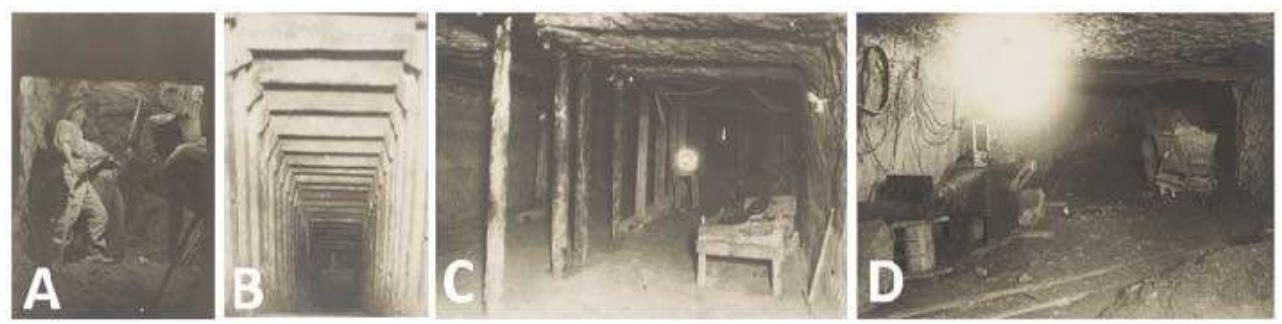

Dans le cadre de la reconstruction de Reims après-guerre, elles ne sont pas réexploitées, mais sont utilisées en champignonnières avant d'être désaffectées, partiellement rebouchées, voire oubliées. Le site est aujourd'hui voué au tourisme sportif, car occupé par un terrain de moto-cross et un spot de parapente.

L'étude de ces creutes d'Hermonville et de leur intégration dans les réseaux de défense repose sur l'analyse spatiale des Plans directeurs des Groupes de Canevas de Tirs des Armées (GCTA) qui permettent de caractériser les réseaux de tranchées et de boyaux des dispositifs de défense français et allemands de 1918 (Combaud et al., 2016). Ces réseaux sont digitalisés sur les feuilles de Reims, Brimont, Berry-au-Bac et Joncherysur-Vesle au 1/20 000 puis traités sous SIG. Pour retrouver les emplacements des entrées des creutes, un travail d'archives est initié, les photographies aériennes de 1929 à 2015 sont interprétées et des campagnes de terrain sont organisées nécessitant des séances de désobstruction.

10 La topographie souterraine des cavités a été réalisée par cheminement à la boussole et au laser-mètre Disto Leica. L'exploration des creutes s'accompagne d'un inventaire des nombreux vestiges de l'occupation militaire à caractère archéologique (mobilier) et pariétal (graffitis, sculptures sur les parois).

11 Une mission lidar aéroportée a été effectuée en décembre 2015 par AERODATA dans le cadre du programme de recherches IMPACT14-18 financé par la région Grand-Est et porté par le GEGENAA. Le traitement des images lidar à haute résolution $(30 \mathrm{~cm}$ en latérale et $3 \mathrm{~cm}$ en altitudinale) de la topographie (13 points $\left./ \mathrm{m}^{2}\right)$ permet de mettre en évidence les micromorphologies associées aux polémo-formes (tranchées, boyaux, entrées d'abris, casemates, trous d'obus) et aux carrières souterraines (bouches de cavage, entonnoirs de soutirage, auges d'affaissement, verses, crevaison de fontis). Les formes révélées par l'image lidar sont reconnues sur le terrain. Le croisement de l'aléa 
cavité et des morphologies de surface permet ainsi de caractériser les désordres d'ordres géotechniques associés aux creutes.

\section{Résultats}

\section{A. Apport des plans directeurs}

L'analyse régionale sous SIG des plans directeurs des GCTA montre que le secteur du Rougement s'inscrit dans la $4{ }^{\mathrm{e}}$ position de défense française. Cette position calée sur la partie sommitale du front de cote dans le secteur de Reims est fondamentale, car elle est la seule garantissant au réseau de défense français, d'une part de magnifiques potentialités pour l'observation (repérage terrestre du dispositif ennemi, réglage des tirs d'artillerie) et d'autre part, des emplacements pour les batteries lourdes. Du côté allemand, les buttes-témoins de Brimont et de Berru assurent cette fonction dans les positions avancées (Devos et al., 2015 ; Taborelli, 2018).

La distance de la cote à la ligne de front (plus de $7 \mathrm{~km}$ ) et la vue dégagée sur l'ensemble de la plaine de Reims garantit l'efficacité des tirs d'artillerie (Fig. 3). Ceci explique donc l'épaisseur exacerbée $(20 \mathrm{~km})$ du dispositif de défense allié dans l'entonnoir de percée cataclinale de la Vesle alors qu'il se rétrécit dans la vallée de la Loivre au nord de Reims $(6 \mathrm{~km})$. L'éperon du Rougemont surveille cette vallée ainsi que la butte de Brimont fortement fortifiée par les troupes allemandes. Il est marqué par des lignes de tranchées discontinues, des boyaux desservant des pièces d'artillerie et relié par des boyaux de liaison avec la 3èmeposition située en contrebas de la cuesta. Les positions avancées sont davantage fortifiées avec une densité accrue de tranchées et de boyaux. La seconde position s'adossant à la route de Cambrai à Reims correspond à la ligne de résistance antérieure à l'offensive du 16 avril 1917. C'est au cours de cette dernière et au prix de lourdes pertes qu'a étéprise la première position allemande. La ligne de front est alors calée sur le canal de l'Aisne à la Marne. 
Document 3 : Bloc-diagramme des réseaux de défense du secteur de Reims (situation fin 1917 début 1918).

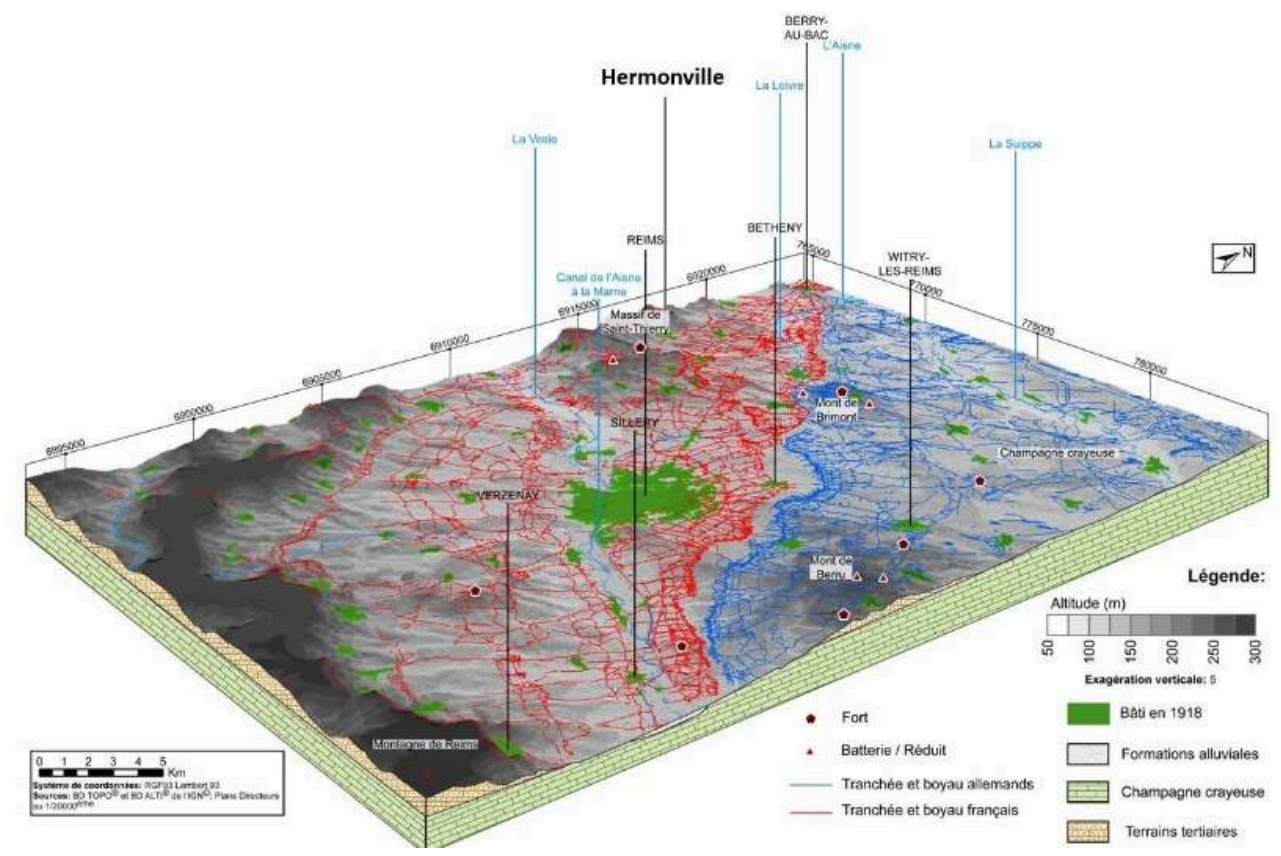

14 À l'échelle locale, au centre de l'éperon du Rougemont, les plans directeurs des groupes de canevas de tirs (Fig. 4) laissent entrevoir la cheminée d'aération «d'anciennes champignonnières » entre le flanc septentrional et le flanc méridional témoignant des forts développements des carrières souterraines. Elles permettent également d'évoquer la forte probabilité de communications entre les nombreuses bouches de cavage (20 à 25) creusées sur les deux flancs de l'éperon (Fig. 4). La tour de l'ancien moulin à vent, utilisée comme observatoire, figure également sur la planimétrie du plan directeur. 
Document 4 : Extrait de la planimétrie des plans directeurs de Berry-au-Bac centré sur le Rougemont (Coll. Personnelle).

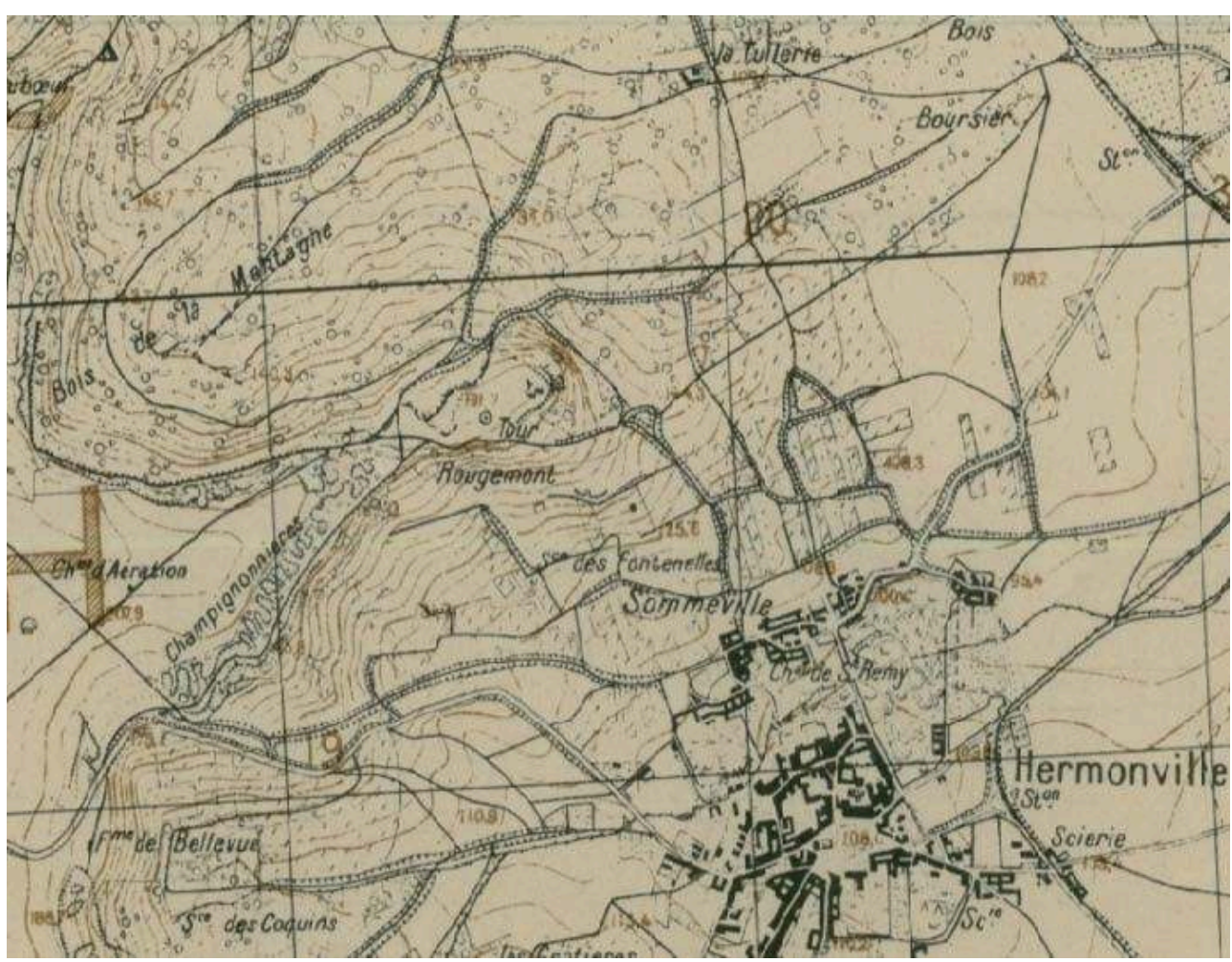

\section{B. Apport de l'approche souterraine}

L'important développement des creutes est confirmé par l'approche souterraine (Fig. 5). En effet, elle témoigne d'une exploitation mixte en piliers tournés, en hagues et bourrages. Le cheminement reconnu y est a minimade $4728 \mathrm{~m}$ (Tab.1) couvrant un espace sous-cavé supérieur à 8 ha (sur les 11 ha que compte l'éperon du Rougemont). Ce chiffre reste sous-estimé, car l'exploration des cavités s'arrête non seulement sur des cônes de fontis, mais surtout sur des hagues avec ou sans bourrages, obstruant l'accès. 3 petites carrières ( $\mathrm{H} 1, \mathrm{H} 2$ et $\mathrm{H} 3)$ à faible développement, sont reconnues sur le flanc nord alors que le flanc sud est davantage percé de galeries dans l'axe des bouches de cavage dont l'intégralité est à ce jour, effondrée ou bouchée.

Document 5 : Image lidar du versant septentrional du Mont-Chatté en 2016

Les carrières souterraines (en rouge) sont desservies par des boyaux et des tranchées de la 4ème position de défense française qui s'organisent autour de sites d'artillerie et d'observation. Les verses des carrières réhabilitées par les militaires drapent le front de la cuesta d'lle de France. 


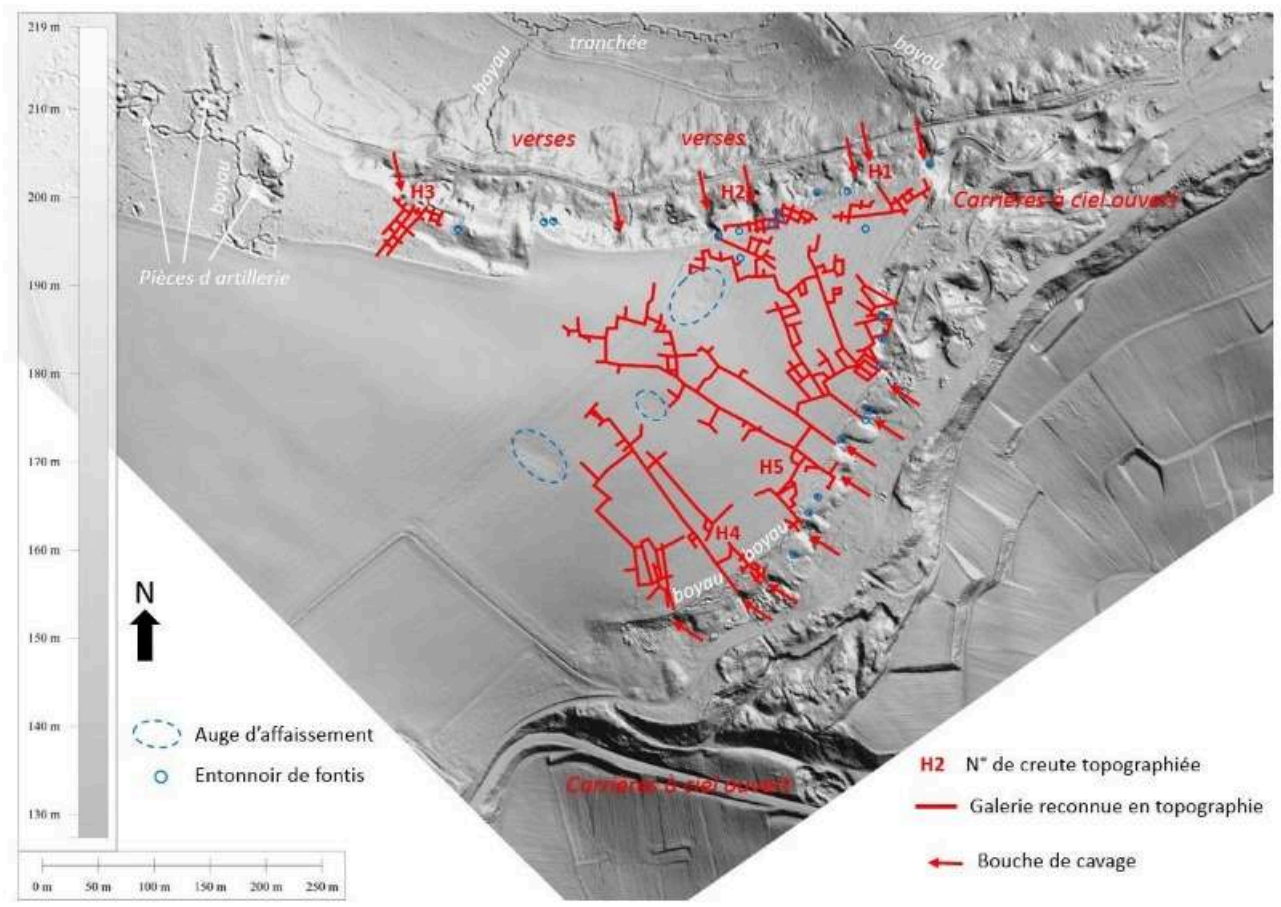

L'exploration des creutes d'Hermonvilletémoigne clairementde l'occupation militaire de ces lieux avec de nombreuses traces, vestiges et mobiliers archéologiques (Fig. 6).

Document 6 : Traces militaires de la vie quotidienne dans les creutes d'Hermonville

18 A. entrée de la creute $\mathrm{H} 2$ avec tôle " métro ", B. tunnel de liaison, C. escalier d'accès aux tranchées, D. lit et bobine de fil téléphonique, E. brodequins, F. toile bituminée sur paroi, G. pot de crème Simon, H. savon-dentifrice GIBBS, I. bouteille alimentaire, J. pot de moutarde, K. tuyau de pipe, L. peigne (cl. : A. Devos).
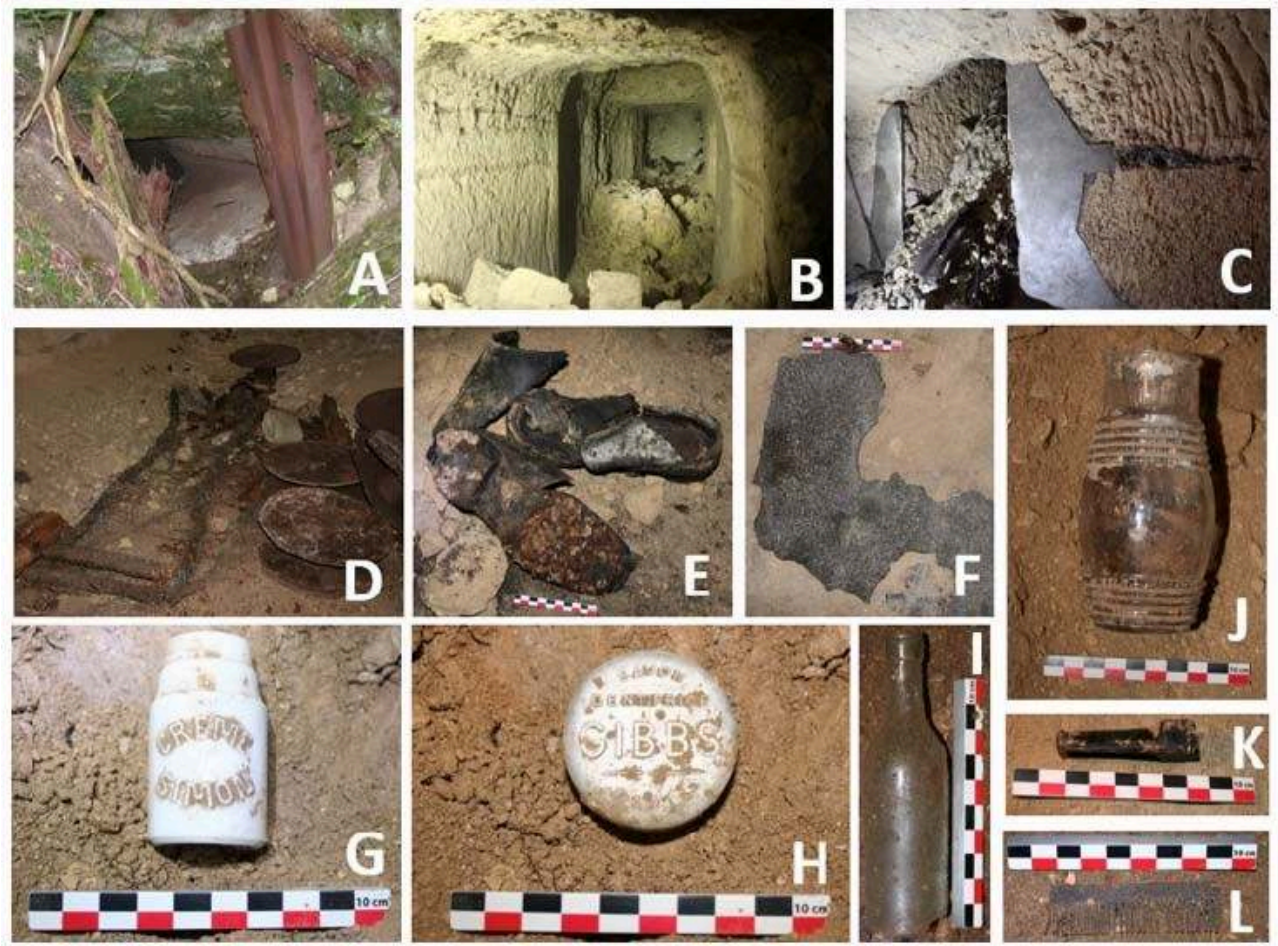

Revue de géographie historique, 14-15 | 2019 

feuillures de portes en béton, piliers construits, piliers à bras). Les remblais des carriers sont creusés pour augmenter l'habitabilité. Les entrées sont aménagées (Fig. 6A), des tunnels de liaison entre secteurs de creutes sont creusés (Fig. 6B) ainsi que des escaliers d'accès aux tranchées (Fig. 6B). Mais les vestiges les plus prégnants sont liés à la vie quotidienne associée au cantonnement dans la quasi-intégralité du réseau prospecté à l'exception de la creute $\mathrm{H} 3$. On retrouve les restes de lits à structure bois et grillage (Fig. 6D), des brodequins (Fig. 6E), des isolateurs en porcelaine, des verres de lampe à huile, des reliquats de toile bitumée fixée aux parois (Fig. 6F), d'anciennes portes et feuillures de portes, de nombreux clous-porte-besace, ainsi que du mobilier militaire et civil agrémentant l'alimentation du quotidien comme des pots de moutarde (Fig. $6 \mathrm{~J}$ ), des flacons en verre alimentaire (Fig. 6I), des objets de toilette (peigne, savon GIBBS, crème SIMON, Fig. 6G, H, L) et du tabac (tuyau de pipe, Fig. 6K). noir de fumée et au crayon de mine, datent pour la plupart de 1917 et correspondent à des insignes militaires (cor de chasse pour les chasseurs, grenade pour l'infanterie), à des patronymes affublés de la date de classe du service militaire et de l'appartenance régimentaire comme le $63^{\text {ème }} \mathrm{BCA}$ (Bataillon de Chasseurs Alpins), une Cie (compagnie) de mitrailleurs du $50^{\text {ème }} \mathrm{BCP}$ (Bataillon de Chasseurs à Pieds), la $6^{\mathrm{e}} \mathrm{Cie}$ du $44^{\mathrm{e}}$, les $51^{\mathrm{e}}$ et $87^{e}$ RI (Régiment d'Infanterie )la Cie $7 / 4$ du Génie, la Cie $2 / 4$ du $3^{e}$ Génie, le $2^{\text {eme }}$ Tirailleur, etc...(Fig. 7).

Document 7 : A.B. insignes du 63e BCA, C. insigne de BCP, D. insigne de bataillon de chasseurs et patronyme du sapeur Houet, E.F. portraits sculptés, G. inscription au noir de fumée de la Compagnie 7/4 du Génie en 1917, H. patronymes de soldats du 51e RI, I. inscription à la mine d'Eugène Landrieu Classe 1911, J. inscription à la mine de Tisserand Elie classe 1906 du 44e (cl. : A. Devos).

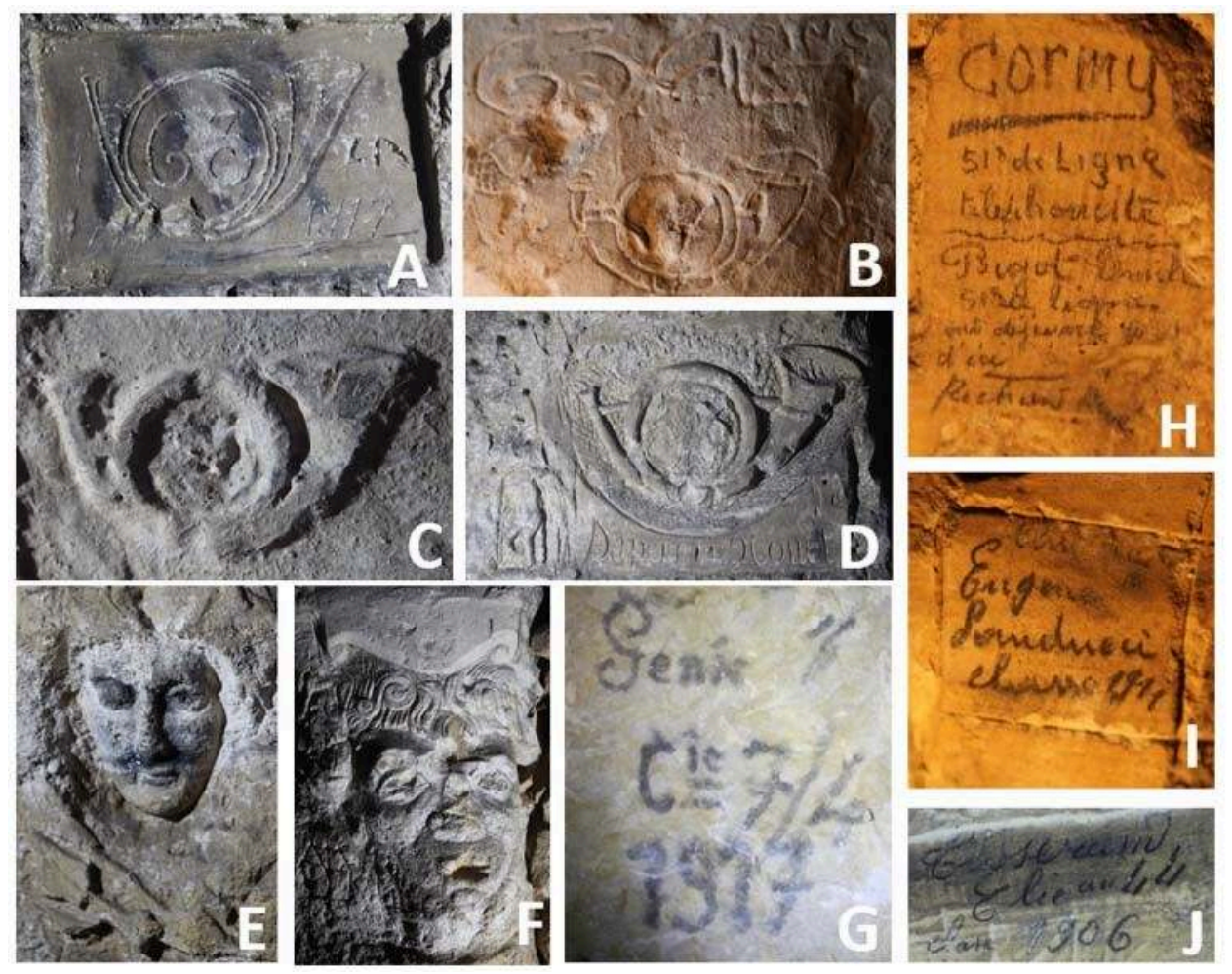

Revue de géographie historique, 14-15 | 2019 


\section{Apport de l'imagerie lidar et des campagnes de terrain}

22 L'image lidar de l'éperon du Rougemont, montre à la fois des morphologies associées à l'exploitation des calcaires lutétiens, des polémo-formes de la Grande Guerre et des modelés postérieurs (Fig. 5 et Fig. 8). Trois niveaux d'exploitation apparaissent dont deux correspondent nettement à des fronts de taille étagés. Il s'agit de celui de la base du Lutétien (calcaires àDitrupa, organisés en dalles décimétriques) et de celui du Lutétien moyen (calcaires coquilliers à milioles et Orbitolites) formant une corniche plus élevée. Le troisième niveau de cavités est moins important et correspond uniquement à quelques creutes d'extension limitées, parfois accompagnées de sapes, creusées sous les premières dalles calcaires du Lutétien et dans la partie sommitale des sables sous-jacents de l'Yprésien supérieur (Sables de Cuise).

Document 8 : Vue lidar 3D du flanc sud de l'éperon du Rougemont avec ses 4 boyaux d'accès aux creutes, reliés à une tranchée en contrebas.

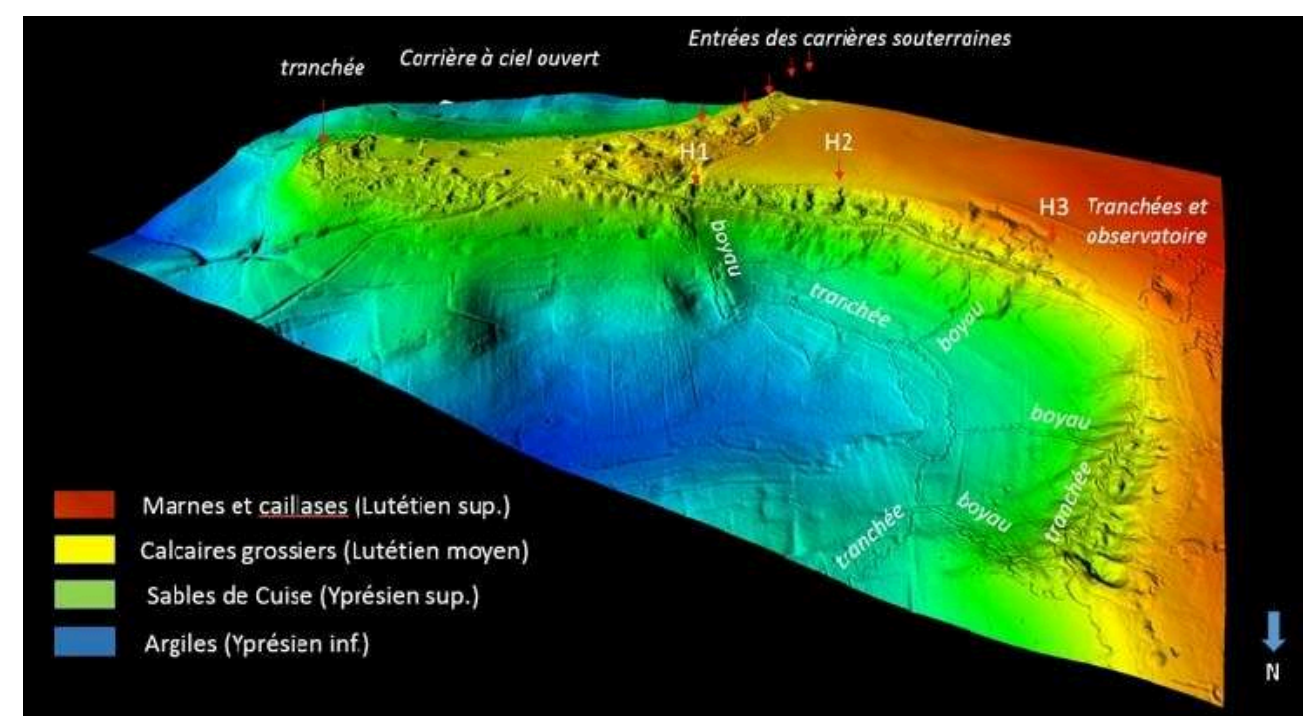

Le premier niveau de carrière est essentiellement exploité dans la terminaison septentrionale de l'éperon, affectée d'un replat intensément creusé par des carrières à ciel ouvert puis relayé par une plateforme périphérique notamment sur le flanc sud, également creusé par des carrières aériennes à la différence du flanc nord.

Le second niveau est exploité par un front de taille aérien qui semble avoir progressivement reculé et par des exploitations souterraines localisées dans le prolongement des carrières aériennes et passant sous les Marnes et caillasses du Lutétien supérieur. Le front de taille aérien est ainsi creusé par 20 à 25 bouches de cavage, désormais souvent comblées, mais qui débouchent sur les galeries reconnues par l'approche souterraine. Le recul du front de taille dégage une plateforme manifestement anthropique (remblais/stériles), bien développée sur le flanc sud (50 à $100 \mathrm{~m}$ de large), et plus réduite sur le flanc nord (moins de $50 \mathrm{~m}$ ) où des verses de carrières drapent sa partie inférieure entre les creutes H1 et H3, sous couverture forestière contrastant ainsi avec le versant opposé, régularisé par les travaux des vignes (Fig. 9). 
Document 9 : Boyau d'accès à la creute H1 (photo : A. Devos), B. observatoire bétonné (photo : JP Batteux).
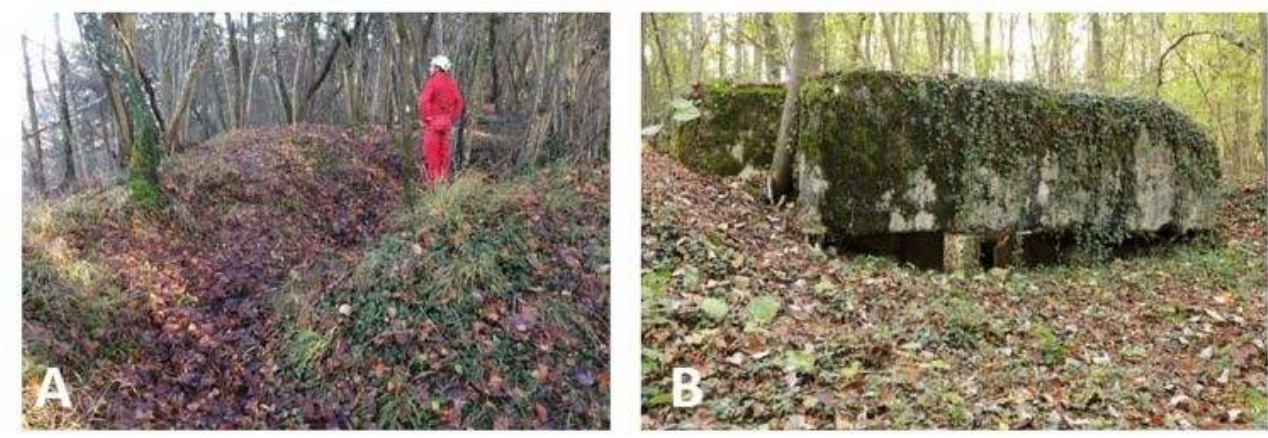

Les morphologies de la Grande Guerre caractérisent surtout le flanc nord avec une tranchée de pied de cote desservant 4 boyaux de liaison vers l'embouchure des entrées des creutes (H1, H3). Une tranchée de surveillance subsiste sur la terminaison septentrionale de l'éperon alors qu'en bordure du plateau, des éléments de tranchée, des abris, des emplacements d'artillerie et un poste d'observation casematé (bunker) sont bien marqués dans le paysage, sous forêt (Fig. 9). Sur le front de cote, une tranchée en créneaux au pied du front de taille assure la liaison entre les entrées des creutes sous couverture forestière, car la piste d'accès est trop exposée à l'ennemi. Des entonnoirs d'obus vraisemblablement associés à des tirs de destruction contre-batterie parsèment le plateau.

D'autres formes témoignent de désordres géotechniques associés aux creutes. Sur le plateau, au centre de l'éperon, 3 auges d'affaissement (dont un à l'aplomb du puits d'aération comblé) jalonnent la surface cultivée. Ces dépressions mesurent de 10 à $20 \mathrm{~m}$ de diamètre, mais sont peu perceptibles à l'œil (Fig. 5 et 10A). En périphérie de l'éperon, sous forêt, on dénombre 22 entonnoirs de fontis (Fig. 10B) d'une profondeur comprise entre 2 et $5 \mathrm{~m}$. Ces dépressions subcirculaires fermées sont associées à des cônes de soutirage des argiles vertes des Marnes et caillasses, àl'aplomb des cheminées des fontis et à des ruptures localisées du toit des galeries observées au fond (Fig. 10C).

Document 10 : Désordres géotechniques associés aux creutes d'Hermonville. A. Auge d'affaissement au centre de l'éperon. B. entonnoir de fontis périphérique. C. cône de soutirage de cheminée de fontis, constitué d'argiles vertes des Marnes et Caillasses observées au fond (cl. : A. Devos). 


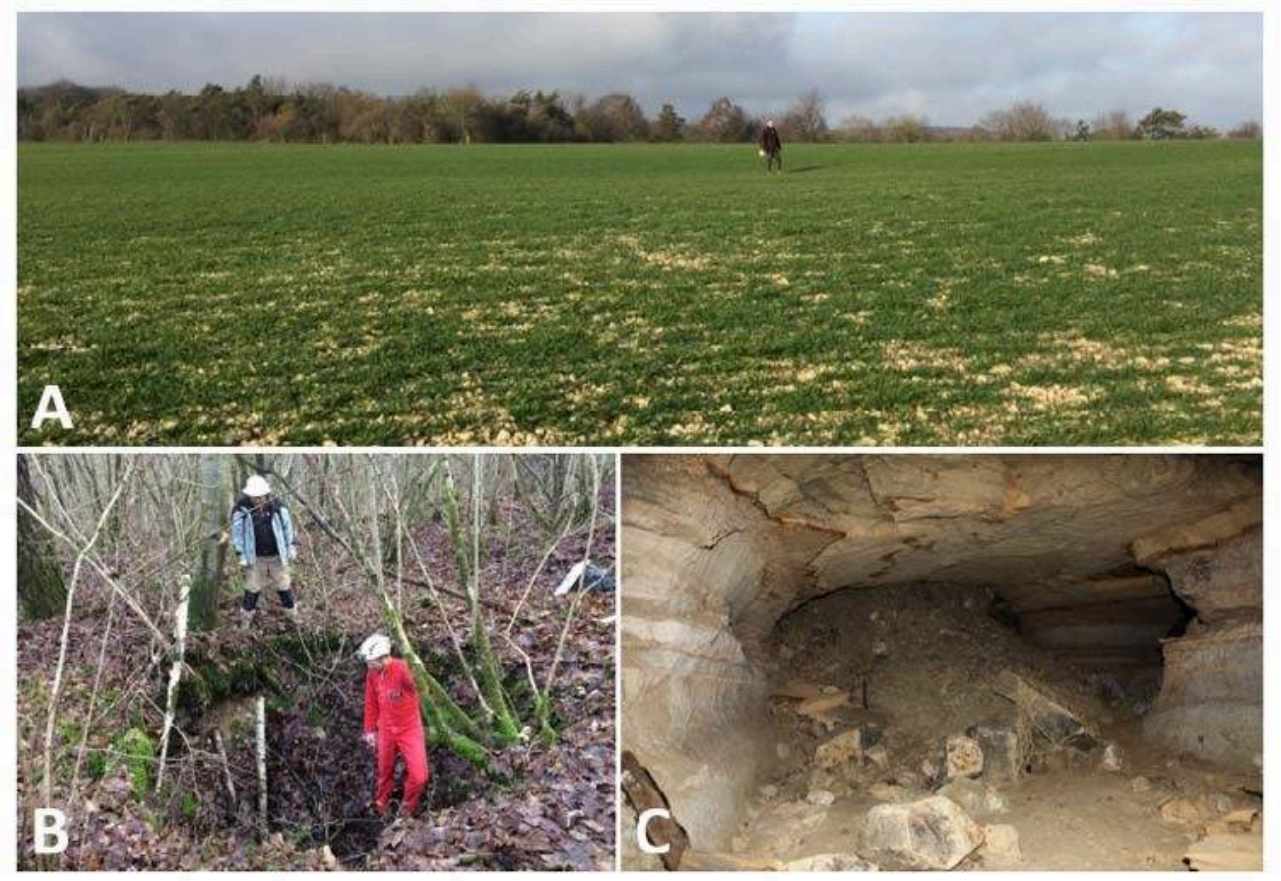

Enfin, les images lidar, complétées par des reconnaissances de terrain, montrent des formes postérieures à la Grande Guerre et associées aux pratiques sportives récentes. Les carrières aériennes et les bouches de cavage sont réhabilitées en parcours de motocross et de trial qui exploitent le modelé hérité des carrières. De nombreux dépôts sauvages de gravats et déchets verts dans les bouches de cavage contribuent aussi, à l'obstruction des creutes notamment sur le flanc sud où toutes les entrées sont à ce jour bouchées.

\section{Discussion}

Si le rôle des creutes est largement discuté par les historiens (Hardier, 2004), un faisceau d'arguments issus du croisement d'approches différentes montre que ces cavités constituent des abris remarquables à l'épreuve des tirs d'artillerie et des intempéries et peuvent être considérées comme essentielles dans la défense des sites (Triolet et Triolet, 2011). L'analyse spatiale des plans directeurs des GCTA sous SIG, les images lidar, l'approche souterraine et les campagnes de terrain en surface, témoignent de l'intégration des creutes d'Hermonville aux réseaux de défense. À l'image des autres creutes du Soissonnais et du Noyonnais (Laforgerie et Weiss, 2013; Weiss, 2016), elles sont englobées dans les réseaux de tranchées et de boyaux et conditionnent même leurs tracés. Les creutes peuvent occuper les premières positions voire se situer dans le no man's land comme la Caverne du Dragon (Devos et Taborelli, 2018 ; Devos et al., 2018; Malinowski, 2004) qui a longtemps cristallisé l'attention voire résumé à elle seule le rôle des creutes au Chemin des Dames en occultant les innombrables autres cavités. En effet, elles sont pour la plupart utilisées en position de réserve, pour le cantonnement et en $4{ }^{\text {ème }}$ rideau comme à Hermonville. Dans le secteur de Reims, elles s'inscrivent en bordure des plateaux tertiaires à $\mathrm{km}$ de la ligne de front, et sont ainsi intégrées aux observatoires d'intérêt régional, bénéficiant du camouflage naturel assuré par la forêt (pinèdes et feuillus) et hébergant les troupes de réserve. 
L'intérêt militaire des creutes est tel, que l'Armée crée en 1916 les bataillons M.D. (Mascart-Dessoliers) du Géniepour les aménager. Ces bataillons sont regroupés le 30 mai 1917, après l'offensive Nivelle, pour leur aménagement dans le Soissonnais à proximité des lignes. Dès le 25 septembre 1917, entre Laffaux et Braye-en-Laonnois, l'Armée dispose d'abris aménagés pouvant héberger plus de 15000 hommes (Protard, 1923). Chaque carrière fait l'objet d'une réhabilitation spatiale et est divisée en quartiers, pour des postes de commandement, magasins (fourrier, munitions...), des ambulances souterraines (Thomas, 2009), des postes de production d'électricité (génératrices), des postes téléphoniques, des dortoirs, des chapelles souterraines (Confrécourt, Rouge Maison, Grotte de l'Eléphant à Soupir, Fig. 1).

31 Dans le secteur de Reims, l'intégration des carrières souterraines et cavités aux réseaux de défense ne se limite pas aux calcaires lutétiens, mais également à la craie. En effet, les crayères, galeries filantes des Maisons de Champagne, souterrains de la Marne, caves privatives, dans le tissu urbain de Reims sont utilisées comme abris entre les deux premières positions de défense française (Devos et al., 2017). Les Allemands font de même en creusant de nombreux tunnels dans les Mont de Champagne (Mont Cornillet, Mont Sans Nom ;Guillaume, 1918). Mais c'est plus particulièrement en Picardie, dans le secteur d'Arras, où l'analyse spatiale, enfin utilisée dans le cadre d'une approche archéologique (Bergerat et al., 2015; Desfossés, et al., 2008), témoigne du rôle fondamental des cavités dans l'organisation spatiale des tranchées. Les carrières médiévales et modernes, en arrière immédiat de la première position britannique servent au cantonnement, et au transfert des troupes vers les premières lignes dans le cadre des offensives de printemps 1917. Mais, elles sont également reliées aux faisceaux de galeries de la guerre des mines.

Devant la valeur militaire opérationnelle des creutes, vérifiée durant la bataille du Chemin des Dames, l'Armée française crée le 30 août 1917 des «bataillons d'attaque des creutes » dans la 6èmearmée. Elle prévoit pour ces unités l'utilisation intensive des gaz et une préparation d'artillerie spécifique. Désormais, dans le cadre de grandes offensives, les entrées des creutes sont la cible de l'artillerie lourde qui envoie de fréquentes rafales d'obus spéciaux. Durant l'offensive française de 1917, les carrières de Montparnasse et leurs abords, occupées par les Allemands, ont reçu, par minute durant quatre heures, vingt coups de $75 \mathrm{~mm}$, dix coups de $155 \mathrm{mmlong}$ et $1 / 2$ coup de $400 \mathrm{mmou}$ de $370 \mathrm{~mm}$ (Corda, 1920) soit un total de 7320 impacts. Ce bombardement a provoqué des effondrements partiels et la démolition des entrées. Les creutes de Frutty, toutes proches, photographiées en reconnaissance aérienne puis au sol après leur prise par les Français, présentent des crevaisons de toit importantes témoignant de l'intensité du bombardement.

33 Cette vulnérabilité apparente des creutes, explique les nombreux cas de troupes piégées par l'effondrement du toit, comme la $3^{\mathrm{e}}$ compagnie du $52^{\mathrm{e}}$ R.I., ensevelie dans une creute de Cuisy, le 16 juin 1917. Le déblaiement se poursuit jusqu'au 18 juin où le bilan fait état de 38 tués, 42 blessés, 2 chevaux tués, 2 cuisines roulantes hors d'usage, une voiture médicale, etc. (JMO, 1917). Le $60^{\mathrm{e}}$ R.I., dont l'état-major, s'est réfugié dans la " grotte du Petit Bois » à la cote 132, le 12 janvier 1915 durant la bataille de Crouy est également affectée par l'effondrement du ciel des carrières. La creute est atteinte par deux fois induisant l'effondrement du toit qui ensevelit plus de soixante hommes. Perdue puis reprise par les Français, la grotte fait l'objet de fouilles en septembre 1917 puis d'une cérémonie le 17 septembre. Dans le secteur de la creute d'Hurtebise, suite à 
son effondrement, «deux compagnies du 18e R.I. ontétéanéanties ou ensevelies et mises dans l'impossibilitéde sortir »(JMO, 1915).

Cependant, les carrières souterraines ont, dans leur ensemble, résisté aux plus violents bombardements. Le plus souvent l'ébranlement des explosions n'a provoqué que le décollement du ciel dans les secteurs d'entrée et là où le recouvrement est peu épais (Devos et Taborelli, 2018).

Si les creutes sont effectivement bien englobées dans la zone de front, leur rôle doit être nuancé selon leur localisation dans les positions de défense que l'analyse spatiale sous SIG à partir des plans directeurs met en évidence. En effet, leur importance dépend non seulement de l'épaisseur du dispositif de défense centré sur la ligne de front (20 à $40 \mathrm{~km})$, et de la densité linéaire et surfacique $(13000 \mathrm{~km}$ de tranchées et de boyaux en Champagne (Taborelli, 2018; Taborelli et al., 2017a), mais aussi des conditions morphostructurales et morpho-dynamiques qui déterminent les potentialités de creusement. L'organisation spatiale des affleurements lutétiens est fonction du degré d'incision des vallées dans les plateaux tertiaires et des variations de puissance du Lutétien, inhérentes aux conditions de dépôt (Gély, 2009). Les potentialités en géoressources sont également dépendantes des dynamiques géomorphologiques et notamment des structures superficielles qui déterminent les contraintes à l'exploitation (Bollot et al., 2014 ; Devos et al., 2012 ; Devos, et al., 2015).

D'est en ouest, de la Montagne de Reims au Soissonnais, le degré d'intégration des creutes est donc variable et tend nettement à augmenter. En effet, à l'est de Reims, en limite de sédimentation du Lutétien, les faciès continentaux et littoraux, les faibles épaisseurs ainsi que les dynamiques géomorphologiques (cambrure, fracturation de détente de versant, extension latérale) sont peu propices à l'exploitation. Ceci explique la faible intégration des cavités à l'exception de la 4èmeposition française (Devos et al., 2015). À l'inverse, à l'ouest de Reims et plus spécifiquement dans le Soissonnais, les faciès marins, la puissance du Lutétien $(40 \mathrm{~m})$ et ses caractéristiques pétrographiques, ainsi que le degré d'incision des vallées offrent de belles potentialités de creusements. L'aquifère perché des calcaires lutétiens, en position d'interfluve présente une zone non saturée suffisamment épaisse, également propice au creusement. Ces paramètres expliquent ainsi la forte densité des creutes et leur bonne intégration dans les réseaux dans toutes les positions de défense.

\section{Conclusion}

Le rôle des cavités souterraines préexistantes dans les réseaux de défense de la Grande Guerre dépend donc conjointement de la géographie de la zone de front, mais aussi des conditions géologiques, hydrogéologiques et géomorphologiques qui conditionnent les potentialités de creusement. Cela implique une prise en compte de ces paramètres et une intégration des géologues dans les Armées durant le conflit qui s'avère très hétérogène selon les Pays belligérants (Ginsburger, 2018).

Si la démarche historique minimise le rôle des carrières souterraines dans la Grande Guerre (Hardier, 2004), l'analyse spatiale des plans directeurs des GCTA croisée à l'outil lidar et à l'approche souterraine sur le Mont Chatté à Hermonville, montre que ces cavités sont englobées dans les réseaux de défense, voire conditionnent leurs tracés. La réhabilitation des creutes par les militaires, induit également des actions morphogènes sur le modelé, associées à des risques géotechniques et pyrotechniques. Cette 
occultation historique s'explique en partie, par la géolocalisation imprécise des cavités, voire ignorée, car elles sont souvent tombées dans l'oubli ou volontairement bouchées par principe de précaution. Ces cavités de la Grande Guerre caractérisées par un patrimoine pariétal exceptionnel, mériteraient d'être référencées dans une banque de données spatialisées sous système d'information géographique, d'une part pour leur préservation et d'autre part pour mieux gérer les risques géotechniques voire pyrotechniques associés.

\section{BIBLIOGRAPHIE}

Amat J.P., 2015, Les forêts de la Grande Guerre : histoire, mémoire, patrimoine, Paris, Presses de l'Université Paris-Sorbonne, 548 p.

Bergerat F., Jacques A., Vandyckes S., Amédro F., Rodaszynski F., Faÿ O., 2015, « Les carrières souterraines d'Arras : géologie, archéologie et histoire », Bulletin d'Information des Géologues du Bassin de Paris, 52, 3, p. 3-26.

Bollot N., Devos A., Pierre G., Chalumeau L., Lejeune O., 2014, « Détente mécanique et dynamique de versant dans l'Eocène de l'est du bassin de Paris, France », Géomorphologie : relief, processus, environnement, 20, vol. 20-n ${ }^{\circ}$ 2, p. 121-132.

Brenot J., Saulière N., Léty C., Taborelli P., Zélie B., Blondeau R., Devos A., 2017, « How much did the soldiers dig ? Àquantification of WW1 remains in Argonne, France », Geoarcheology.

Combaud A., Devos A., Chalumeau L., Taborelli P., Bollot N., 2016, « Les plans directeurs de la Grande Guerre : Une nécessaire innovation cartographique » Davoine P.-A., Beck E. (dir.), Revue Internationale de Géomatique, 26, 3, p. 363-393.

Corda H., 1920, La bataille et son évolution pendant la Guerre 1914-1918. Cours de tactique, 1ère et 2nd divisions, École Militaire de l'Artillerie, Paris, 117 p.

Desfossés Y., Jacques A., Prilaux G., 2008, L'archéologie de la Grande Guerre, Rennes, Ouest-France/ INRAP, $127 \mathrm{p}$.

Devos A., Bollot N., Chalumeau L., Fronteau G., Lejeune O., 2015, «Impact of lateral variations of geologic facies on water resources in homogeneous basins: example of tertiary plateaus in the Paris Basin », Geodinamica Acta, 27, 1, p. 15-24.

Devos A., Chalumeau L., Bollot N., Sosson C., Barre E., Lejeune O., Gobancé G., 2012, « Organisation spatiale de la fracturation de détente mécanique de versant en carrières souterraines.

Application aux plateaux des calcaires éocènes du Bassin de Paris ", Physio-Géo, 6, 1, p. 243-262.

Devos A., Taborelli P., 2018, «L'utilisation des souterrains : creutes et cagnas du Soissonnais et du Pays Rémois », dans Bergerat F. (dir.), 14-18, La Terre et le Feu. Géologie et géologues sur le front occidental, co-édition AGBP-COFRHIGÉO-SGN, Paris, p. 68-81.

Devos A., Taborelli P., Damien T., Bollot N., Blanc A., Fronteau G., Laratte S., Lejeune O., Desfosses Y., Embry M., 2017, « La Grande Guerre sur la Cote d'Île-de-France, en Champagne crayeuse et en Argonne - Journées d'étude d'Automne - World War I in Ile-de-France cuesta, Chalky 
Champagne and Argonne- Days of autumn study - », bulletin Information des Géologues du bassin de Paris, 54, 3, p. 3-19.

Devos A., Taborelli P., Desfossés Y., 2018, «L'organisation spatiale du front : une question d'échelle ! », dans Bergerat F. (dir.), 14-18, La Terre et le Feu. Géologie et géologues sur le front occidental, co-édition AGBP-COFRHIGÉO-SGN, Paris, p. 68-81.

Devos A., Taborelli P., Dodici M., Chalumeau L., Buridant J., Bollot N., Combaud A., Desfossés Y., 2015, «Rôle des conditions géographiques sur l'organisation spatiale des réseaux de défense de la Grande Guerre. Application à la Champagne (région de Reims) », Physio-Géo, 9, 1, p. 157-174.

Dolfini L., 1920, « Recherches de Carrières. Reims ", Rapport du Ministère de l'Instruction Publique et des Beaux-Arts, Architecte en chef. Mr Deneux.

Fronteau G., Devos A., Bouvy C., Chalumeau L., Tomachot-Schneider C., Turmel A., Lejeune O., Decrocq B., 2014, « Recherche d'un équivalent à la Pierre de Courville (Lutétien, Marne, France) pour les chantiers de restauration de la cathédrale Notre-Dame de Reims », rapport final, DRAC Champagne-Ardenne.

Fronteau G., Turmel A., Pichard C., Decrock B., Devos A., Lejeune O., Ménival D., Chalumeau L., Combaud A., 2014, « Les approvisionnements en pierre de construction à Reims : des choix marqués par de fortes contraintes géologiques, géographiques et socio-économiques ", dans Construire la ville. Histoire urbaine de la pierre à bâtir.

Gély J.-P., 2009, « LE LUTÉTIEN : une période charnière de l'histoire du Bassin parisien »,Saga Information, 284, p. 6-24.

Ginsburger N., 2018, « Géologues et géologie de guerre pendant et après la Première Guerre mondiale : heurs et malheurs d'une science mobilisée ", dans Bergerat F. (dir.), 14-18, La Terre et le Feu. Géologie et géologues sur le front occidental, co-édition AGBP-COFRHIGÉO-SGN, (Mém. hors-série $\mathrm{n}^{\circ} 10$ de l'AGBP), p. 291-301.

Guillaume C., 1918, « Note du 22 septembre 1918 du Chef de Bataillon Guillaume commandant les unités du génie de la 163 ème DI ».

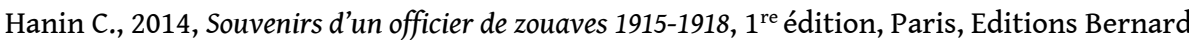
Giovanangeli, $270 \mathrm{p}$.

Hardier T., 2004, « Une guerre souterraine, creutes et tunnels du Chemin des Dames ", dansoffenstadt N. (dir.), Le Chemin des Dames - de l'évènement à la mémoire, Stock, p. 493.

JMO, 1915, JMO de la 36ème DI, 26 janvier 1915.

JMO, 1917, JMO du 52ème RI, 16 juin 1917.

Lachaux G., 2005, Les Creutes : Chemin des Dames et Soissonnais, L'encrier du Poilu. Cerneux (Seineet-Marne), $292 \mathrm{p}$.

Lachaux G., 2008, Chemin des Dames - L'album souvenir du front de l'Aisne, Histoire \& Collections, Paris, $195 \mathrm{p}$.

Laforgerie E., Weiss J.-F., 2013, Les souterrains de la Première Guerre mondiale - du creusement au témoignage, Ysec, $223 \mathrm{p}$.

Malinowski A., 2004, Le Chemin des Dames - La caverne du Dragon, Ysec, Louviers, 240 p.

Prilaux G., Bossut D., 2018, Graffitis \& bas-reliefs de la Grande Guerre : archives souterraines de combattants, Paris, INRAP : Michalon, 146 p. 
Protard G., 1923, « Les bataillons M.D., Revue du génie militaire 1923/01-1923/06, (janv-juin 1923) », Ed. Berger-Levrault, tome LII, p. pp 309-358 et pp.405-438.

Sosson C., Devos A., Lejeune O., Fronteau G., 2006, « Les“creutes” : carrières souterraines entre Reims, Laon et Soissons », Subterranea, 138, p. 50-62.

Taborelli P., 2018, Les conditions géographiques et l'organisation spatiale du front de la Grande Guerre : application à l'évaluation environnementale post-conflit en Champagne-Ardenne (France),Thèse de doctorat, Reims, France, Université de Reims-Champagne-Ardennes, 425 p., 425 p.

Taborelli P., Devos A., Laratte S., Brenot J., Cancès B., Desfossés Y., 2017a, « Apport des plans directeurs et de l'outil LiDAR aéroporté pour la caractérisation des impacts morphologiques de la Grande Guerre : exemple de la cote 108 (Berry-au-Bac, France) ", Géomorphologie : relief, processus, environnement, 23 , vol. $23-\mathrm{n}^{\circ} 2$.

Taborelli P., Devos A., Laratte S., Desfossés Y., Brenot J., 2017b, « Typologie et organisation spatiale des « polémo-formes » de la Grande Guerre révélées par l'outil LiDAR et les Plans directeurs. Application à la Champagne et à l'Argonne », Revue de Géographie Historique 10-11.

Thomas G., 2009, «Quand le service de santé faisait “carrières”. La réutilisation des creutes de l'Aisne pour l'activité du Service de santé, pendant le premier conflit mondial. », Médecine et Armées, 37, 3, p. 283-288.

Triolet J., Triolet L., 2011, La guerre souterraine - sous terre, on se bat aussi, Paris, Perrin, 348 p.

Villate R., 1925, Les conditions géographiques de la guerre sur le front français de 1914 à 1918, Paris, Payot (Bibliothèque géographique), $325 \mathrm{p}$.

Weiss J.-F., 2016, Les souterrains de la Première Guerre mondiale - du creusement au témoignage, AREESVS, $223 \mathrm{p}$.

\section{RÉSUMÉS}

L'approche spatiale de la Grande Guerre à partir des plans directeurs montre que sur le front ouest, marqué par la guerre de position, les réseaux de défense structurés en positions et en lignes de tranchées, s'organisent selon les potentialités et contraintes naturelles (reliefs, eau, masque de la végétation) et les logiques militaires (crêtes de tirs, sites d'observation). Les carrières souterraines du Soissonnais (creutes) et du nord-ouest de Reims, creusées dans les Calcaires grossiers du Lutétien moyen sont englobées dans les réseaux de défense. Les militaires aménagent les carrières pour le cantonnement (ventilation, électrification, dégagement des remblais), creusent des tunnels de liaison en s'adaptant à la karstification, à la fracturation et aux écoulements qui existent dans l'encaissant calcaire. Dans le secteur du Mont Chatté (Hermonville), ces actions morphogènes sont observées sous terre et révélées par l'image lidar aéroporté (verses de carrières sur le front de cuesta, entonnoirs de soutirage associés aux cavités, boyaux d'accès aux bouches de cavage, etc.). Poilus, Tommies et soldats allemands produisent de très nombreux graffitis, sculptures et traces pariétales qui constituent un patrimoine souterrain original.

The spatial approach of the Great War from the directing plans shows that on the western front, characterized by trench warfare, the defense networks structured in positions and in trench lines, are organized according to natural potentialities and constraints (reliefs, water, mask of vegetation) and military logics (cannon range, observation sites). The underground quarries of Soissonnais (creutes) and north-west of Reims, dug in the coarse limestones of the Middle Lutetian are included in the defense networks. The soldiers set up the quarries for the 
cantonment (ventilation, electrification, clearance of embankments), dig tunnels of connection by adapting to the karstification, the fracturing and the flows which exist in the limestone country. In the Mont Chatté (Hermonville) sector, these morphogenic actions are observed underground and revealed by the airborne Lidar image (quarries on the cuesta front, filler funnels associated with the cavities, communication trench to the quarries entrance, etc ...) .Poilus, Tommies and German soldiers produce many graffiti, sculptures and parietal traces that constitute an original underground heritage.

\section{INDEX}

Mots-clés : Grande Guerre, réseaux de défense, carrières souterraines, Lutétien, lidar, Hermonville

Keywords : Great War, defense network, underground quarries, Lutetian, lidar, Hermonville, France

\section{AUTEURS}

\section{ALAIN DEVOS}

57 rue P. Taittinger, BP 30, 51571 REIMS Cedex ; alain.devos@univ-reims.fr

\section{SÉBASTIEN LARATTE}

2, rue Pierre Richer-de-Belleval, 51100 CHALONS-EN-CHAMPAGNE., lesjean.frank@marne.fr

NICOLAS BOLLOT

GEGENAA-URCA, 2 esplanade Roland Garros, 51100 REIMS

G. FRONTEAU

GEGENAA-URCA, 2 esplanade Roland Garros, 51100 REIMS 